\title{
Comparative study of two local anesthetics in the surgical extraction of mandibular third molars: Bupivacaine and articaine
}

\author{
Anna Trullenque-Eriksson ${ }^{1}$, Blanca Guisado-Moya ${ }^{2}$ \\ ${ }^{1}$ Bachelor of Dentistry. Master in Dentistry Science. Universidad Complutense de Madrid \\ ${ }^{2}$ Professor of Oral Surgery, Department of Oral and Facial Medicine and Surgery. Faculty of Dentistry. Universidad Complu- \\ tense de Madrid
}

Correspondence:

Faculty of Dentistry

Plaza Ramón y Cajal s/n.

Ciudad Universitaria

28040 Madrid

anna@trullenque.com

Received: $15 / 06 / 2010$

Accepted: $14 / 11 / 2010$
Trullenque-Eriksson A, Guisado-Moya B. Comparative study of two local anesthetics in the surgical extraction of mandibular third molars: Bupivacaine and articaine. Med Oral Patol Oral Cir Bucal. 2011 May 1;16 (3):e390-6.

http://www.medicinaoral.com/medoralfree01/v16i3/medoralv16i3p390.pdf

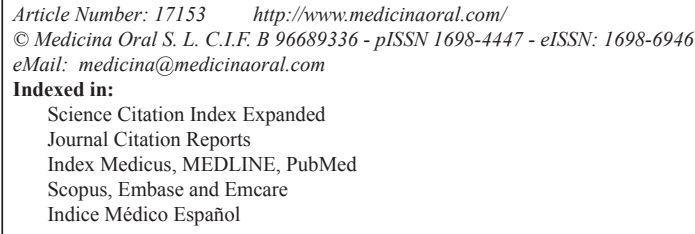

Indice Médico Español

\begin{abstract}
Objective: The third molar extraction is one of the most common surgical procedures in oral surgery and is usually accompanied by postoperative discomfort. It has been suggested that the longer duration of action of bupivacaine associated with the residual analgesia and the gradual onset of pain, could decrease the need for analgesics during the postoperative period. This study aims to compare the efficacy and safety of bupivacaine and articaine as local anesthetics for the extraction of mandibular third molars and to check whether bupivacaine produced residual analgesia.

Study design: We compared bupivacaine $0.5 \%$ and articaine $4 \%$ with an epinephrine concentration of 1:200 000 in a crossover design model of extraction of bilaterally symmetrical mandibular third molars.

Results: Regarding efficacy, patients experienced less postoperative pain at 6 and 12 hours and shorter duration of soft tissue anesthesia with articaine. With respect to safety, no differences were found between the anesthetics compared, showing a similar local and systemic toxicity. With regard to the preference of patients, it was higher for articaine, the main reasons being the greater postoperative pain and swelling with bupivacaine.

Conclusion: It can be concluded that articaine seems to be a more appropriate anesthetic for the extraction of mandibular third molars due to the shorter duration of the anesthetic effect in the soft tissues, lower pain reported by patients during the immediate postoperative period and the personal preference of patients for this drug.
\end{abstract}

Key words: Anesthesia, dentistry, bupivacaine, articaine, oral surgery. 


\section{Introduction}

Local anesthetics are chemicals that block nerve conduction in a specific, temporary and reversible manner, without affecting the patient's consciousness. The molecule consists of two poles: a hydrophilic tertiary or secondary amino group and a lipophilic aromatic ring. According to the type of intermediate alkyl linkage between them, they are classified in ester-type anesthetics, with an amino-ester bond and whose prototype is procaine, and the amide-type with an amino-amide bond and whose prototype is lidocaine (1).

Until the beginning of the twentieth century, cocaine was the drug of choice for surgical and dental pain control despite its significant limitations such as its low therapeutic index, the risk of addiction and potentially lethal arrhythmias. After its synthesis in 1904 by Alfred Einhorn procaine became the main local anesthetic in medicine and dentistry. Because of the long latency period of procaine and allergies to ester anesthetics, lidocaine, the first amide anesthetic, quickly became the gold standard after its synthesis in 1943 by Nils Löfgren. Other amide anesthetics have subsequently been introduced $(2,3)$.

Bupivacaine was developed in 1957 by Ekenstam, Egner and Pettersson. Its clinical use was described by Widman in 1964, and in 1983 it became commercially available in dental cartridges. It is a powerful amide anesthetic, with intermediate onset of action and long duration, allowing a slow return to normal sensation, which has been associated with a corresponding gradual onset of pain (Table 1) (1-6).

It has been suggested that long-acting local anesthetics such as bupivacaine, could provide additional analgesia time known as "residual analgesia" and minimize the duration of postoperative pain, facilitating postoperative care and maintenance of proper oral hygiene. Its main indications are lengthy procedures and postoperative pain management $(5,7,8)$.
Carticaine was synthesized in 1969 by Rusching et al., changing its name to articaine in 1976, when it was introduced in German dentistry. It was first used in Canada in 1983, the United Kingdom in 1998 and in the United States in 2000. It is an amide anesthetic that contains a thiophene ring, being the only widely used anesthetic that contains an ester group. It is a powerful anesthetic, with rapid onset of action and intermediate duration (Table 1) $(2,9,10)$.

Some professionals claim that its effect is quicker, is associated with few failures and provides anesthesia in cases where other anesthetics fail. However, there is little scientific evidence to demonstrate its superiority over other local anesthetics. Its use in oral surgery is very common (2).

Because of its diverse pathology, third molar extraction is one of the most common surgical procedures in oral surgery, and is generally accompanied by postoperative discomfort, leading to the intake of analgesics, anti-inflammatories and antibiotics, and sometimes absenteeism from work. It has been suggested that the longer duration of action of bupivacaine associated with the residual analgesia and the gradual onset of pain, may reduce the need for analgesics, which could be interesting given the side effects that these are associated with $(3-5,11-13)$.

This study aims to compare the efficacy and safety of bupivacaine and articaine as local anesthetics for the extraction of mandibular third molars, and verify whether bupivacaine produced residual analgesia. The following hypotheses were tested:

- Bupivacaine has a greater efficacy than articaine when used as an anesthetic for extraction of mandibular third molars, providing a period of residual analgesia, which reduces the need for analgesics during the postoperative period.

- Bupivacaine is as safe as articaine when used as an an-

Table 1. Characteristics of bupivacaine and articaine.

$\begin{array}{lll} & \text { Bupivacaine } & \\ \text { Chemical structure } & \text { Amide } & \text { Articaine } \\ \text { Potency } & \text { High } & \text { Amide with ester group (thiophene ring) } \\ \text { Onset } & \text { Intermediate } & \text { High } \\ \text { Duration of action } & \text { Long } & \text { Rapid } \\ \text { Side effects } & \text { Cardiotoxic } & \text { Moderate }\end{array}$


esthetic for the extraction of mandibular third molars. The following secondary objectives were established: - Within the comparison of the efficacy, collect information on postoperative pain and inflammation.

- Within the comparison of safety, measuring patients' vital signs and bleeding during surgery, as well as verifying the postoperative complications possibly related to these drugs.

- Assess the patient's preference for one or the other local anesthetic.

\section{Materials and Methods}

In this study we compared bupivacaine $0.5 \%$ and articaine $4 \%$, both with an epinephrine concentration of 1:200,000 in a crossover design model of extraction of bilaterally symmetrical mandibular third molars.

Patients were selected from among those who attended the surgical department of the Faculty of Dentistry, Universidad Complutense de Madrid, for the extraction of bilateral mandibular third molars between October 2008 and March 2009. We carried out a non-probabilistic sampling of consecutive cases and selected 35 patients ASA I or ASA II.

We included those patients who were to undergo extraction of bilaterally symmetrical mandibular third molars when both were of similar surgical difficulty and similar estimated duration of the intervention. If the patient also required upper third molar extraction, these had to be of similar surgical difficulty and estimated duration of the intervention. In the subsequent analysis, only the mandibular molars were taken into account. Patients had to sign a consent form for surgery and inclusion in the study.

We excluded patients who had allergy or hypersensitivity to the local anesthetics, antibiotics or analgesics used, pregnant patients, patients with cardiovascular, liver or renal disease, hyperthyroidism, diabetes mellitus, immunosuppression or chronic pain, and patients who had taken drugs (except oral contraceptives) .

Each patient underwent two surgical procedures, performed by the same surgeon and separated a minimum of two weeks. Anesthesia was administered by blocking the inferior alveolar nerve, accompanied by lingual and buccal nerve block with carpule-type syringes and anesthetic in dental cartridges. The manufacturer's instructions were followed for the administration of the anesthetic, and the dose was the one that the surgeon deemed necessary for surgery, usually two cartridges, one for the anesthesia of the inferior alveolar and lingual nerves, and one for the buccal nerve. The patients were randomly administered one of the two local anesthetics in the first surgery, and the other one in the following. The anesthetic used was unknown for the patient and the observer who performed the measurements. At the time of the surgery this information was only known by the surgeon who administered the anesthesia and the surgeon who assisted him, who recorded the anesthetic and dose in the patient's medical history and a collection sheet in an opaque envelope, which were not consulted until the data analysis. After the surgical procedure, all patients were prescribed the same antibiotic (750mg amoxicillin every 8 hours for 7 days) and antiinflammatory (600mg ibuprofen, maximum once every 6 hours in case of pain).

The study variables were measured and collected during surgery, as well as in the postsurgical and final questionnaires.

To evaluate the efficacy, we assessed those variables that gave information about the action of the anesthetic, both those measured during surgery (time of onset, need for reinforcement), and the data collected in the following questionnaire (duration of soft tissue anesthesia, duration of residual analgesia, postoperative pain, analgesic intake and decrease in mouth opening) (Table 2).

For the evaluation of safety, we analyzed the patients' vital signs measured with a blood pressure monitor and a pulse oximeter, bleeding during surgery and postoperative complications reported by patients (Table 2).

We evaluated patient preference for either anesthetic (Table 2).

\section{-Statistical analysis}

For the statistical analysis the following analyses were performed with SPSS:

- T-test to compare quantitative variables in related samples, when criteria of normal distribution were met.

- Wilcoxon signed-rank test to compare quantitative variables in related samples where the distribution did not meet criteria of normal distribution.

- Pearson's correlation coefficient to assess the correlation between surgical time and postoperative pain.

- McNemar's test for comparison of the need for reinforcement.

The results are given as mean \pm standard deviation.

\section{Results}

Of the thirty-five patients selected, nineteen were included in the study. The mean age was 24.47 years, with $31.6 \%$ male and $68.4 \%$ women.

In the evaluation of the efficacy of the anesthetics, no statistically significant differences were found for time of onset (inferior alveolar nerve: bupivacaine $3.68 \pm$ 3.11 minutes and articaine $2.81 \pm 1.92$ minutes; buccal nerve: bupivacaine $1.95 \pm 1.25$ minutes and articaine $1.63 \pm 1.14$ minutes), the need for reinforcement (articaine $47.4 \%$ and bupivacaine $31.6 \%$ ), the duration of residual analgesia (bupivacaine $5.11 \pm 4.45$ hours and articaine $4.61 \pm 3.77$ hours), analgesic intake or decrease in mouth opening.

Statistically significant differences were found between the duration of soft tissue anesthesia, this being $8.20 \pm$ 
Table 2. Characteristics of bupivacaine and articaine.

\begin{tabular}{|c|c|c|}
\hline \multirow{7}{*}{ 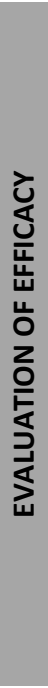 } & Time of onset & $\begin{array}{l}\text { Time between anesthetic administration and the onset of anesthesia: } \\
\text { - Inferior alveolar nerve: lip numbness. } \\
\text { buccal nerve: sensitivity in the vestibular gum innervated by the } \\
\text { buccal nerve. }\end{array}$ \\
\hline & Need for reinforcement & Need to administrate additional anesthesia. \\
\hline & $\begin{array}{l}\text { Duration of soft tissue } \\
\text { anesthesia }\end{array}$ & $\begin{array}{l}\text { Time between onset of anesthesia and stop of soft tissue numbness and } \\
\text { paresthesia. }\end{array}$ \\
\hline & $\begin{array}{l}\text { Duration of residual } \\
\text { analgesia }\end{array}$ & $\begin{array}{l}\text { Time between onset of anesthesia and onset of pain (information obtained } \\
\text { in the postoperative questionnaire). }\end{array}$ \\
\hline & Postoperative pain & Assessment with visual analogue scale (VAS) at 6, 12, 24 and 48 hours. \\
\hline & Analgesic intake & $\begin{array}{l}\text { Number of doses of analgesic and time when they were taken during the } \\
\text { postoperative week. }\end{array}$ \\
\hline & Decrease in mouth opening & $\begin{array}{l}\text { Distance between incisal edge of upper incisors and incisal edge of lower } \\
\text { incisors at maximum opening. Difference between measurement before } \\
\text { surgery and one week after surgery. }\end{array}$ \\
\hline \multirow{3}{*}{ 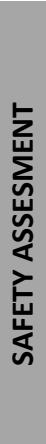 } & Measurement of vital signs & $\begin{array}{l}\text { Before anesthesia, after anesthesia and after surgery: } \\
\text { - Blood pressure: with digital wrist blood pressure monitor (AIRIS } \\
\text { TSO01). } \\
\text { - Pulse and oxygen saturation: with a pulse oximeter } \\
\left.\text { (OhmedaTuffSat }{ }^{\oplus}\right) \text {. }\end{array}$ \\
\hline & Bleeding during surgery & $\begin{array}{l}\text { Subjective scale } 0-3 \text {, with } 0 \text { being the absence of bleeding, } 1 \text { mild bleeding, } \\
2 \text { moderate bleeding and } 3 \text { heavy bleeding. From November } 2008 \text {. }\end{array}$ \\
\hline & Postoperativecomplications & $\begin{array}{l}\text { Postoperative discomforts considered related with surgery or anesthesia by } \\
\text { the patient. }\end{array}$ \\
\hline 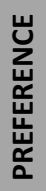 & Preference & Patient described and compared his experience with both anesthetics. \\
\hline
\end{tabular}

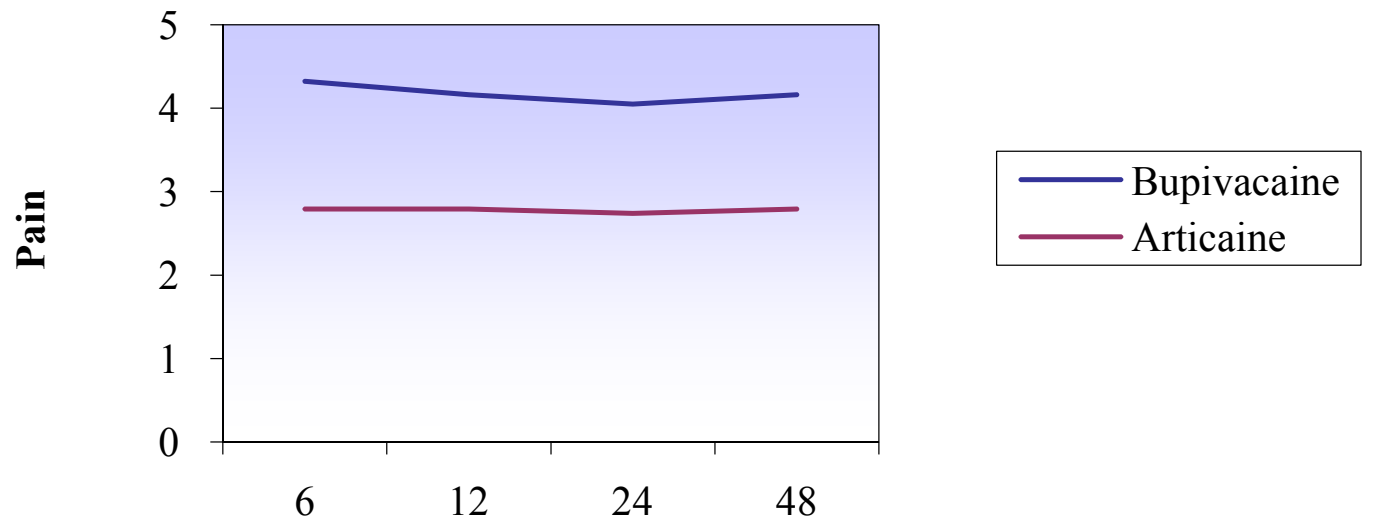

Hours

Fig. 1. Postoperative pain with bupivacaine and articaine. 
4.54 hours in the case of bupivacaine, and $5.32 \pm 2.16$ hours in the case of articaine.

Postoperative pain was significantly lower at 6 and 12 hours in procedures performed with articaine as anesthetic (Fig. 1). In addition it was found that there was a correlation between pain and surgical time for bupivacaine at $6,12,24$ and 48 hours, but not for articaine.

Within the safety assessment of the anesthetics, no statistically significant differences were found for blood pressure, pulse or bleeding during surgery.

For the oxygen saturation, the only significant differences were found in the initial and final measurements, but not between the ones made after the administration of anesthesia or changes in oxygen saturation.

Regarding postoperative complications reported by patients in the postoperative questionnaires, $42.1 \%$ of patients considered to have had at least one. Among the complications reported by patients after the surgery performed with bupivacaine as an anesthetic, were postoperative swelling, infection and bleeding, cramps in the hemimandible where the surgical procedure was performed, headache, bruising, pain at the injection site, dizziness and vomiting. In the case of articaine the complications reported were postoperative swelling, infection and bleeding, ulcers, heat sensation, temporomandibular joint pain, lip droop and sleepiness. It gave the same percentage of patients without postoperative complications, patients with infection, inflammation and bleeding after the surgery for both anesthetics.

Within the patient preference, $63.2 \%$ of the patients chose articaine, $26.3 \%$ bupivacaine and $10.5 \%$ neither. Those who chose the surgical procedure carried out with articaine as the least painful gave as main reasons for this choice the more postoperative pain and swelling after the interventions with bupivacaine.

\section{Discussion}

As the extraction of third molars is very frequent in oral surgery and postoperative discomfort is usually associated, it would be interesting to have an anesthetic that produced residual analgesia for some time, reducing the consumption of analgesics and improving the patient's postoperative experience. Several authors have suggested that bupivacaine could achieve this effect (3-5, 11-13).

The study followed a bilateral crossover design, which has the advantage that each patient acts as his own control. Thus, the individual characteristics of each patient and their subjective assessment of pain do not influence the results of the study. The double-blind contributed to avoid bias, as the observer and the patient ignored the anesthetic used in each surgery. To avoid differences between surgeons and their subjective assessment of bleeding influencing the data collected, the same surgeon performed both interventions in each pa- tient. Likewise, efforts were made for the time between surgeries to be the least possible to make it easier for the patient to compare the interventions and prevent the increasing experience of surgeons from influencing the study results.

To date, we only have knowledge of another study that compares bupivacaine $0.5 \%$ with 1:200 000 epinephrine, with articaine 4\% with 1:200 000 epinephrine in oral surgery (14).

For the evaluation of efficacy we took into account the time of onset, the need for reinforcement, the duration of soft tissue anesthesia, the duration of residual analgesia, postoperative pain, analgesic intake and decrease in mouth opening.

In our study, the time of onset of bupivacaine was greater than that of articaine, but the differences were not statistically significant. However, Gregorio et al. (14) did find statistically significant differences.

Regarding the administration of reinforcement, although it was necessary more frequently in the interventions with articaine, the difference was not significant. In the study by Gregorio et al. (14), reinforcement was necessary in $14 \%$ of surgeries performed with bupivacaine and $2 \%$ of those performed with articaine, this difference was significant. It has been shown that bupivacaine is less effective in infiltrative techniques, due to its high lipid solubility which also makes the position of the needle key $(4,6,11)$. This could explain the difference found in the work by Gregorio et al. (14). The high percentage of interventions that needed additional anesthesia in our study may be more related to anatomical variations or anesthetic technique since the surgery was performed by postgraduate students and not by experienced surgeons, than with the anesthetics.

The values of duration of soft tissue anesthesia reported for bupivacaine were similar to those of other authors, whereas the values for articaine were somewhat higher (Table 3). The differences were statistically significant, like those found by Gregorio et al. (14). The longer duration of soft tissue anesthesia of bupivacaine is explained by its higher protein binding and pKa of 8.1 (6). The longer duration of the anesthesia could be unpleasant for patients and could cause difficulty eating, speaking, and a greater risk of soft tissue trauma $(11,16,17)$.

The duration of residual analgesia was higher for bupivacaine, although the difference was not statistically significant. The study of Gregorio et al. (14) obtained similar results.

Several authors have advocated for the analgesic effect of bupivacaine, with consequent lower perception of pain and less analgesic intake by the patient, compared with other anesthetics such as lidocaine or mepivacaine $(3,5,11,12)$. However, in our study, postoperative pain scores were higher for bupivacaine than for articaine, and this difference was statistically significant at 6 and 
Table 3. Comparisons between $4 \%$ articaíne and $0.5 \%$ bupivacaine, both with epinephrine 1:200 000 .

\begin{tabular}{|c|c|c|c|c|c|c|}
\hline \multirow{2}{*}{ Authors } & \multicolumn{2}{|c|}{$\begin{array}{c}\text { Onset } \\
\text { (minutes) }\end{array}$} & \multicolumn{2}{|c|}{$\begin{array}{l}\text { Duration of soft tissue anesthesia } \\
\text { (hours) }\end{array}$} & \multicolumn{2}{|c|}{$\begin{array}{l}\text { Duration of analgesia } \\
\text { (hours) }\end{array}$} \\
\hline & Articaine & Bupivacaine & Articaine & Bupivacaine & Articaine & Bupivacaine \\
\hline Crout et al. 1990(15) & - & $4.3 \pm 1.0$ & - & $6.82 \pm 0.6$ & - & $5.38 \pm 0.78$ \\
\hline Fernandez et al. 2005 (11) & - & $6.53 \pm 0.68$ & - & $8.22 \pm 0.31$ & - & - \\
\hline Volpato et al. 2005 (7) & - & $14(2-36)$ & - & 9.77 (7.62 - 14.17) & - & - \\
\hline Malamed 2006 (2) & $2-3$ & $6-10$ & $3-5$ & $3-12$ & - & - \\
\hline Gregorio et al. 2008 (14) & $1.66 \pm 0.13$ & $2.51 \pm 0.21$ & 4 & 5 & 3 & 4 \\
\hline
\end{tabular}

12 hours. Another interesting fact was that, while the postoperative pain was correlated with surgical time in procedures where bupivacaine was used as local anesthetic, this did not happen with articaine. The reason for this finding is unknown, although it could be due to pharmacodynamic factors specific to the anesthetic.

Despite the difference in pain at 6 and 12 hours, as in the study of Gregorio et al. (14) no statistically significant differences were found between the time elapsed until analgesic intake or the number of doses.

Regarding decrease in mouth opening, although it was lower after surgical procedures performed with bupivacaine, the difference was not statistically significant. Gregorio et al. (14) found a significant difference when osteotomy was necessary, with a greater decrease in the case of bupivacaine. When they compared all surgeries the decrease was greater in the case of articaine although this difference was not significant.

Therefore, taking into account that patients experienced less postoperative pain at 6 and 12 hours and shorter duration of soft tissue anesthesia in procedures performed with articaine as local anesthetic, articaine appears to be a more appropriate anesthetic for the extraction of mandibular third molars than bupivacaine.

For the safety assessment of the anesthetic we considered the values and changes in blood pressure, pulse and oxygen saturation, bleeding during surgery and postoperative complications.

Although it has been argued that bupivacaine exerts a dose-dependent decrease in systolic blood pressure and articaine exerts an increase in systolic blood pressure $(18,19)$, no significant differences in blood pressure or pulse were found. Gregorio et al. (14) did find significant differences in diastolic blood pressure during the proceedings in which osteotomy was necessary, this be- ing lower in the case of articaine. Regarding the oxygen saturation, the differences found were in the initial and final measurements, which were probably not related to the anesthetic used.

Both local anesthetics having the same concentration of adrenaline, and bupivacaine being more vasodilator than articaine, one might assume that the bleeding during surgery would be greater with bupivacaine. However, as in the study of Gregorio et al. (14), differences in bleeding were not statistically significant.

The postoperative complications reported by the patients in this study are similar to those described elsewhere $(10,14)$. The percentage of patients reporting one or more postoperative complications was high, compared with that obtained by other authors $(10,12,14$, $16,20)$. However, in our study we particularly stressed that patients should describe any postoperative discomfort considered abnormal. Many of those problems are probably not attributable to the anesthetic itself, but to the surgical trauma. Even so, we specified all possible complications that the patient considered related to the anesthesia and the surgery.

There has been some concern about the possibility that local anesthetics at a concentration of $4 \%$ as is articaine could lead to paresthesia, although there is no clear scientific evidence that demonstrates this (2). During the study period there was a case of paresthesia in one patient operated with articaine as a local anesthetic, but this patient was not included in the study due to not attending the surgery on the contralateral side.

It follows from our results that there are no differences between the anesthetics compared, showing a similar local and systemic toxicity.

Regarding the preference of the patients, it was markedly higher for articaine. 
Therefore, even with the limitations of a small sample due to the loss of patients after the first surgery, we can conclude that although there are no differences in terms of safety, the anesthetic articaine seems to be more appropriate for the extraction of mandibular third molars due to the shorter duration of the soft tissue anesthesia, lower pain reported by patients during the immediate postoperative period and the personal preference of patients for this drug.

It might be interesting to carry out studies with a larger number of cases in which the data were analyzed by groups according to the times in different surgical stages.

\section{References}

References with links to Crossref - DOI

1. Milam SB, Giovannitti JA Jr. Local anesthetics in dental practice. Dent Clin North Am. 1984;28:493-508.

2. Malamed SF. Local anesthetics: dentistry's most important drugs, clinical update 2006. J Calif Dent Assoc. 2006;34:971-6.

3. Kumar R, Rao SN. Local anaesthetic for minor oral surgical procedures. Review. Indian J Dent Res. 2000;11:163-6.

4. Sisk AL. Long-acting local anesthetics in dentistry. Anesth Prog. 1992;39:53-60.

5. Chapman PJ, Macleod AW. A clinical study of bupivacaine for mandibular anesthesia in oral surgery. Anesth Prog. 1985;32:69-72.

6. Danielsson K, Evers H, Nordenram A. Long-acting local anesthetics in oral surgery: an experimental evaluation of bupivacaine and etidocaine for oral infiltration anesthesia. Anesth Prog. 1985;32:65-8.

7. Volpato MC, Ranali J, Ramacciato JC, de Oliveira PC, Ambrosano GM, Groppo FC. Anesthetic efficacy of bupivacaine solutions in inferior alveolar nerve block. Anesth Prog. 2005;52:132-5.

8. Moore PA, Nahouraii HS, Zovko JG, Wisniewski SR. Dental therapeutic practice patterns in the U.S. I. Anesthesia and sedation. Gen Dent. 2006;54:92-8.

9. Malamed SF, Gagnon S, Leblanc D. Efficacy of articaine: a new amide local anesthetic. J Am Dent Assoc. 2000;131:635-42.

10. Malamed SF, Gagnon S, Leblanc D. Articaine hydrochloride: a study of the safety of a new amide local anesthetic. J Am Dent Assoc. 2001;132:177-85.

11. Fernandez C, Reader A, Beck M, Nusstein J. A prospective, randomized, double-blind comparison of bupivacaine and lidocaine for inferior alveolar nerve blocks. J Endod. 2005;31:499-503.

12. Trieger N, Gillen GH. Bupivacaine anesthesia and post-operative analgesia in oral surgery. Anesth Prog. 1979;26:20-3.

13. Nayyar MS, Yates C. Bupivacaine as pre-emptive analgesia in third molar surgery: Randomised controlled trial. Br J Oral Maxillofac Surg. 2006;44:501-3.

14. Gregorio LV, Giglio FP, Sakai VT, Modena KC, Colombini BL, Calvo AM, et al. A comparison of the clinical anesthetic efficacy of $4 \%$ articaine and $0.5 \%$ bupivacaine (both with 1:200,000 epinephrine) for lower third molar removal. Oral Surg Oral Med

Oral Pathol Oral Radiol Endod. 2008;106:19-28.

15. Crout RJ, Koraido G, Moore PA. A clinical trial of long-acting local anesthetics for periodontal surgery. Anesth Prog. 1990;37:194-8. 16. Rosenquist JB, Nystrom E. Long-acting analgesic or long-acting local anesthetic in controlling immediate postoperative pain after lower third molar surgery. Anesth Prog. 1987;34:6-9.

17. Mellor DJ, Mellor AH, McAteer EM. Local anaesthetic infiltration for surgical exodontia of third molar teeth: a double-blind study comparing bupivacaine infiltration with i.v. ketorolac. Br J Anaesth. 1998:81:511-4.

18. Younessi OJ, Punnia-Moorthy A. Cardiovascular effects of bupivacaine and the role of this agent in preemptive dental analgesia. Anesth Prog. 1999;46:56-62.

19. Vasconcellos RJ, Vasconcelos BC, Genú PR. Influence of local anesthethics with adrenalina 1:100.000 in basic vital constants during third molar surgery. Med Oral Patol Oral Cir Bucal. 2008;13:E431-7. 20. Rosenquist JB, Rosenquist KI, Lee PK. Comparison between lidocaine and bupivacaine as local anesthetics with diflunisal for postoperative pain control after lower third molar surgery. Anesth Prog. 1988;35:1-4. 